\title{
Relation taxonomique entre Gendria Baylis. 1930 et Cucullanus Mueller, 1777
}

\author{
(Remarques sur la superfamille Seuratoidea Chabaud,
}

Campana-Rouget et Brygoo, 1959)

par LE-VAN-HOA et PHAM-NGOC-KHUE

Institut Pasteur du Viet-Nam

Laboratoire de Microbiologie Animale de l'Institut Pasteur, 167, rue Pasteur, Sä̈gon, Sud-Vietnam.

(Directeur Général: $\mathrm{P}^{\mathrm{r}}$ NGUYEN-VAN-AI).

\begin{abstract}
Résumé
Description de Gendria rauschi n. sp., nouvelle espèce parasite de Rana tigrina et Chitwoodia chitwoodae n. g. n. sp. parasite du poisson d'eau douce Puntius bramoides au Sud Viêt-nam.

En nous basant sur la morphologie de ces parasites et sur celle de Campanarougetia campanarougetae Lê-văn-Hoà et coll., 1967, nous essayons d'établir une lignée Gendria - Chitwoodia - Campanarougetia - Cucullanus.

Cette étude comparative permet de préciser la place des Cucullanus parmi les Seuratoidea Chabaud, Campana et Brygoo 1959. Inglis a raison d'écrire «The Cucullanidae is referred to the superfamily Seuratoidea, Ascaridida $»$.
\end{abstract}

\section{Summary}

Description of Gendria rauschi n. sp., new species of Rana tigrina and Chitwoodia chitwoodae n. g. n. sp., parasite of the freshwater fish Puntius bramoides in South Viet-nam.

The new genus Chitwoodia shows affinity to the Ascaroids by the presence of lip-pulps and allows us to establish a line Gendria - Chitwoodia - Campanarougetia - Cucullanus. 
The above comparative study explains better now the cephalic structure of the Cucullanus. We agree with Inglis 1967, that \& The family Cucullanidae is referred to the Seuratoidea: Ascaridida».

\section{Introduction.}

Le genre Cucullanus, Mueller 1777 a été longtemps classé provisoirement dans les Spirurides par la symétrie bilatérale de la bouche.

Cependant, Yorke et Maplestone (1926) signalent que l'œsophage ressemble plutôt au type d'Ascaride. Chabaud et ses collaborateurs (1960) considèrent les Cucullanidae comme des formes de passage entre Ascarides primitifs (Cosmocercoidea) et des Spirurides.

Les travaux plus récents, réalisés d'une part par Inglis (1967) et d'autre part par Lê-van-Hoà et Pham-ngoc-Khuê (1967) ont permis de mieux comprendre ces nématodes et de les rattacher à la superfamille Seuratoidea, Chabaud, Campana et Brygoo, 1960.

Dans cette note, grâce à deux nouveaux nématodes Gendria rauschi n. sp. et Chitwoodia chitwoodae n. g. n. sp., nous essayons d'établir une ligne Gendria-ChitwoodiaCampanarougetia-Cucullanus. Celle-ci rend encore plus compréhensible et plus naturelle la systématique des Cucullanus.

\section{1. - Gendria rauschi n. sp.}

HôTE : Rana tigrina var rugulosa.

LOCALISATION : intestin.

Matériel : 3 mâles, 5 femelles.

Provenance: Marché de Saïgon, le 6-4-1966.

Description: Nématode de petite taille, muni d'une vésicule céphalique et de deux ailes latérales qui s'effacent au niveau des diérides (fig. 1, A et B). Bouche hexagonale entourée de 6 papilles du cycle interne. Cycle externe formé de 2 amphides latérales et 4 papilles sub-médianes, simples et pédonculées (fig. 1, C). Capsule buccale faible mais chitinoide, portant 3 grosses dents, 2 subventrales simples et une dorsale plus développée et bifide antérieurement (fig. 1, C). Esophage claviforme, divisé en 2 parties : l'antérieur musculaire et le postérieur musculo-glandulaire (fig. 1, A). L'anneau nerveux se situe à la limite des 2 parties de l'œsophage, les diérides au niveau de la terminaison œsophagienne et le pore excréteur plus en arrière (fig. 1, A).

Mâle: Corps long de 11,5-12 mm. Specimen type long de $12 \mathrm{~mm}$ et large de $0,3 \mathrm{~mm}$. Esophage total : 0,620 $\mathrm{mm}$ (partie antérieure : 0,250 $\mathrm{mm}$ sur $0,05 \mathrm{~mm}$; partie postérieure : $0,370 \mathrm{~mm}$ sur $0,09 \mathrm{~mm}$ ). Anneau nerveux, diérides et pore excréteur res- 
pectivement à $0,24 \mathrm{~mm}$, à $0,52 \mathrm{~mm}$ et à $0,6 \mathrm{~mm}$ de l'apex. Vésicule céphalique longue de $0,08 \mathrm{~mm}$. Ailes latérales : $0,43 \mathrm{~mm}$ sur $0,02 \mathrm{~mm}$.

Extrémité postérieure légèrement recourbée ventralement et munie de 9 paires de papilles caudales réparties en 6 préanales, 3 postanales. Il existe également une papille
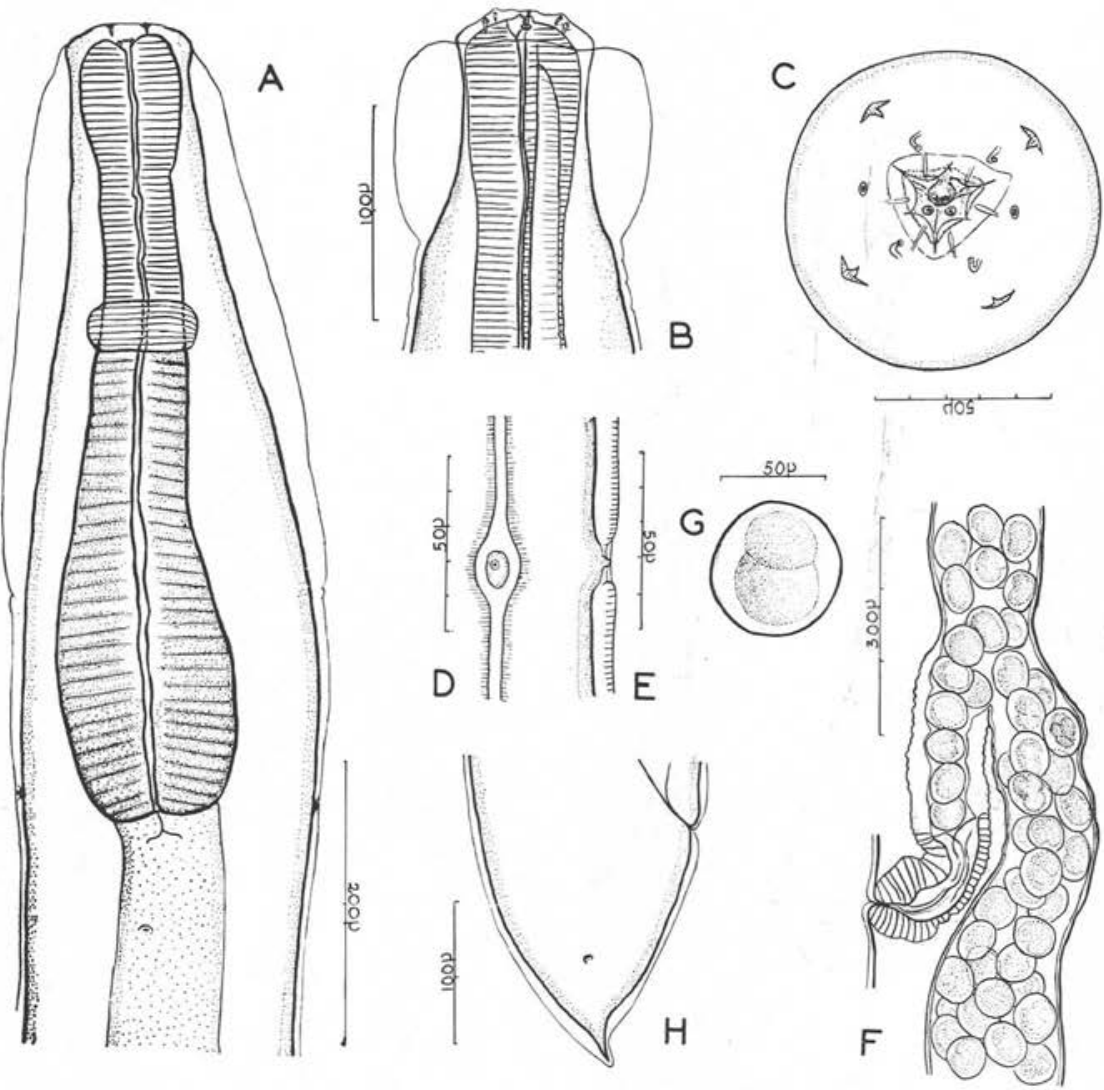

FIG. 1. - Gendria rauschi n. sp. Femelle de $16,5 \mathrm{~mm}$ : A. Extrémité antérieure, vue ventrale, B. Extrémité céphalique, vue latérale; C. Extrémité céphalique, vue apicale; D et E. Diérides; F. Région vulvaire, vue latérale; G. CEuf; H. Queue, vue latérale

médiane en avant du cloaque et 2 phasmides (fig. 2, A et B). Ventouse précloacale allongée, relativement peu développée, mesurant $0,1 \mathrm{~mm}$ sur $0,035 \mathrm{~mm}$. Distance entre le centre de la ventouse et l'ouverture cloacale : $0,47 \mathrm{~mm}$. Spicules subégaux, ailés et soutenus par 2 tiges chitinoides. Le spicule droit atteint $0,38 \mathrm{~mm}$ sur $0,05 \mathrm{~mm}$, le gauche $0,36 \mathrm{~mm}$ sur $0,05 \mathrm{~mm}$ (fig. 2, C et D). Gubernaculum long de $0,04 \mathrm{~mm}$ (fig. 2, E et F). Queue longue de $0,14 \mathrm{~mm}$. 
Femelle: Specimen type long de $16,5 \mathrm{~mm}$ et large de 0,4 $\mathrm{mm}$. Esophage total : $0,58 \mathrm{~mm}$ (partie antérieure $0,23 \mathrm{~mm}$ sur $0,05 \mathrm{~mm}$; partie postérieure 0,33 sur $0,125 \mathrm{~mm}$ ). Anneau nerveux, diérides et pore excréteur respectivement à $0,21 \mathrm{~mm}$, à $0,56 \mathrm{~mm}$ et à $0,64 \mathrm{~mm}$ de l'apex. Vésicule céphalique longue de $0,09 \mathrm{~mm}$. Ailes laté rales : $0,54 \mathrm{~mm}$ sur $0,03 \mathrm{~mm}$.
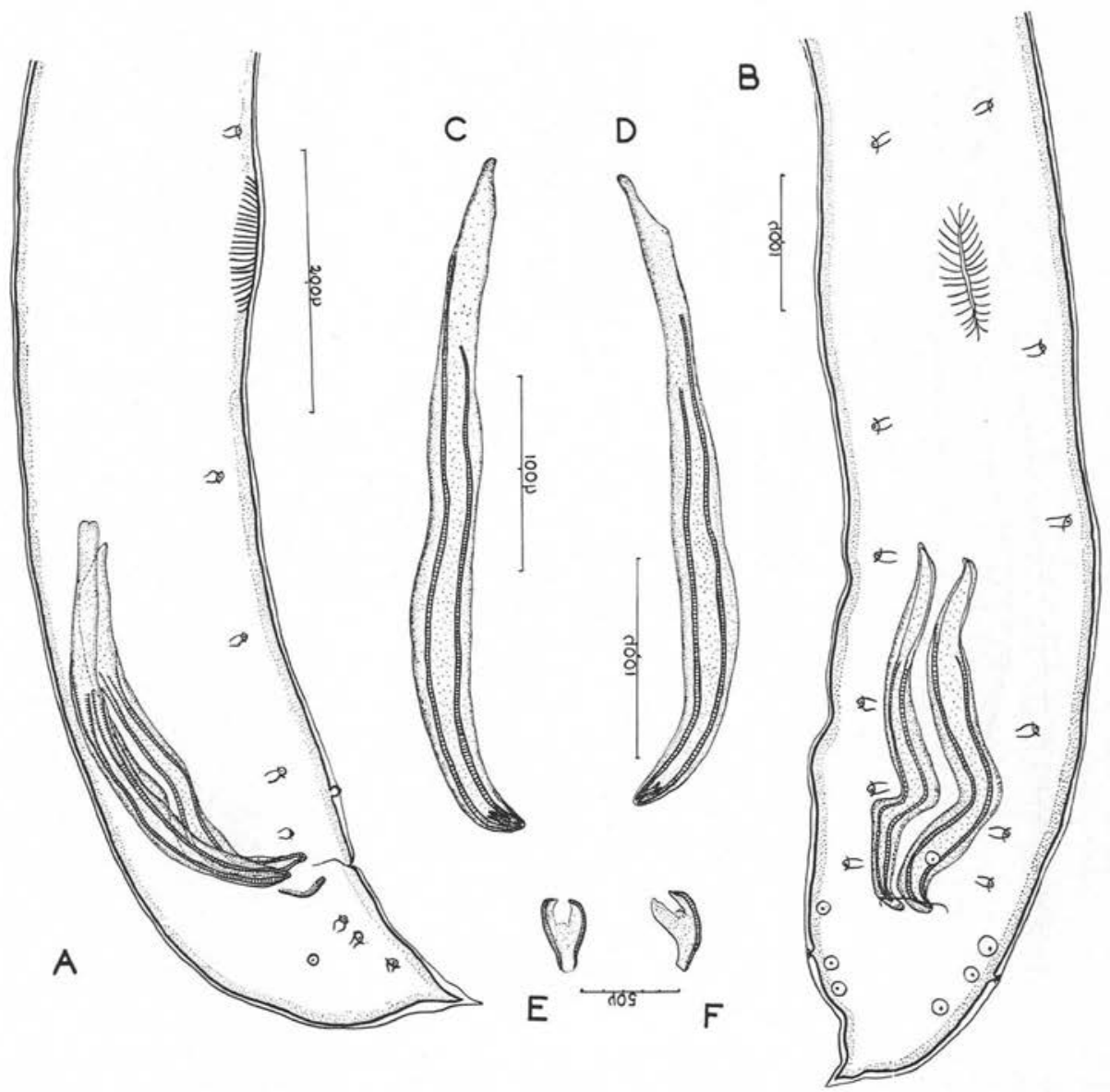

Fig. 2. - Gendria rauschi n. sp. Mâle de $12 \mathrm{~mm}$ : A. Extrémité postérieure, vue latérale ; B. Extrémité postérieure, vue ventrale ; C. Spicule droit ; D. Spicule gauche; E et F. Gubernaculum

Vulve non saillante située à $8,8 \mathrm{~mm}$ de l'extrémité postérieure. Le vagin musculeux, dirigé en avant, mesure $0,2 \mathrm{~mm}$ sur $0,1 \mathrm{~mm}$. Le tronc commun de l'utérus, long de $0,23 \mathrm{~mm}$, se divise en deux branches divergentes (fig. 1, F). Eufs non embryonnés, subsphériques, à coque mince. Diamètre des œufs : 0,065-0,068 mm sur 0,060-0,062 mm (fig. 1, G). Queue longue de 0,175 mm (fig. 1, H). 
Discussion: Nos parasites ont quelques caractères aberrants d'un Metaquimperia, Karve, 1941, par la présence des ailes latérales et d'un œsophage divisé en 2 parties. Malgré cela, nous préférons les ranger dans Gendria, Baylis, 1930, en se basant sur la vésicule céphalique et les 3 grosses dents pharyngiennes.

Parmi les espèces du genre, nous avons à comparer avec les espèces suivantes :

- Gendria tilapiae, Baylis, 1930, parasite de Tilapia galilaea au Niger, G. brevispiculum, Karve, 1941, chez Macrones sp. et G. ranarum, Karve, 1944, chez Rana tigrina aux Indes, qui se distinguent de nos spécimens par leur œsophage simple et plus court.

- Gendria leberrei, Bain et Philippon, 1969, chez Bufo regularis en Haute Volta, paraît plus proche de nos nématodes mais n'a pas d'ailes latérales; la dent dorsale est simple, non bifide; des spicules brusquement atténués aux extrémités distales.

Nous proposons de nommer la nouvelle espèce Gendria rauschi n. sp. en l'honneur du $D^{r}$ Robert-L. Rausch.

\section{2. - Chitwoodia chitwoodae n. g. n. sp.}

Hôte : Puntius bramoides.

LOCALISATION : intestin.

MATÉrIEL : 2 mâles, 2 femelles et 2 femelles immatures.

Provenance : Marché de Saïgon, le 11-10-1969.

DESCRIPTION: Corps relativement trapu, mais effilé à son extrémité postérieure. Bouche entourée de 3 lèvres, réduites et soudées à leurs bords latéraux.

Chaque lèvre est munie de deux pulpes labiales atrophiées évoquant celles des ascarides (Fig. 3 B). Système papillaire externe composé de 2 amphides et 4 papilles submédianes simples et pédonculées.

Capsule buccale réduite, chitinoide et pourvue de 3 petites dents (fig. 3,C). Esophage simple, court, trapu et dilaté antérieurement (fig. 3, D et E).

Mâle : Corps long de $9,75 \mathrm{~mm}$ et large de $0,4 \mathrm{~mm}$. Esophage long de $0,57 \mathrm{~mm}$ et large de $0,22 \mathrm{~mm}$ au renflement antérieur, de $0,12 \mathrm{~mm}$ au niveau de l'anneau nerveux et de $0,18 \mathrm{~mm}$ au renflement postérieur. Anneau nerveux, diérides et pore excréteur respectivement à $0,38 \mathrm{~mm}$, à $0,84 \mathrm{~mm}$ et à $0,9 \mathrm{~mm}$ de l'apex.

Extrémité postérieure légèrement recourbée ventralement. Ailes caudales étroites, longues de $0,52 \mathrm{~mm}$. Il existe 13 paires de papilles caudales: 6 paires préanales, 1 adanale et 6 paires postanales. La deuxième paire postanale est en position latérale (fig. 4, $\mathrm{E}$ et F). Spicules sclérifiés et subégaux, le droit long de $0,21 \mathrm{~mm}$, large de $0,02 \mathrm{~mm}$, le gauche long de 0,2 mm, large de 0,02 mm (fig. 4, D). Gubernaculum absent. Queue longue de $0,3 \mathrm{~mm}$. 

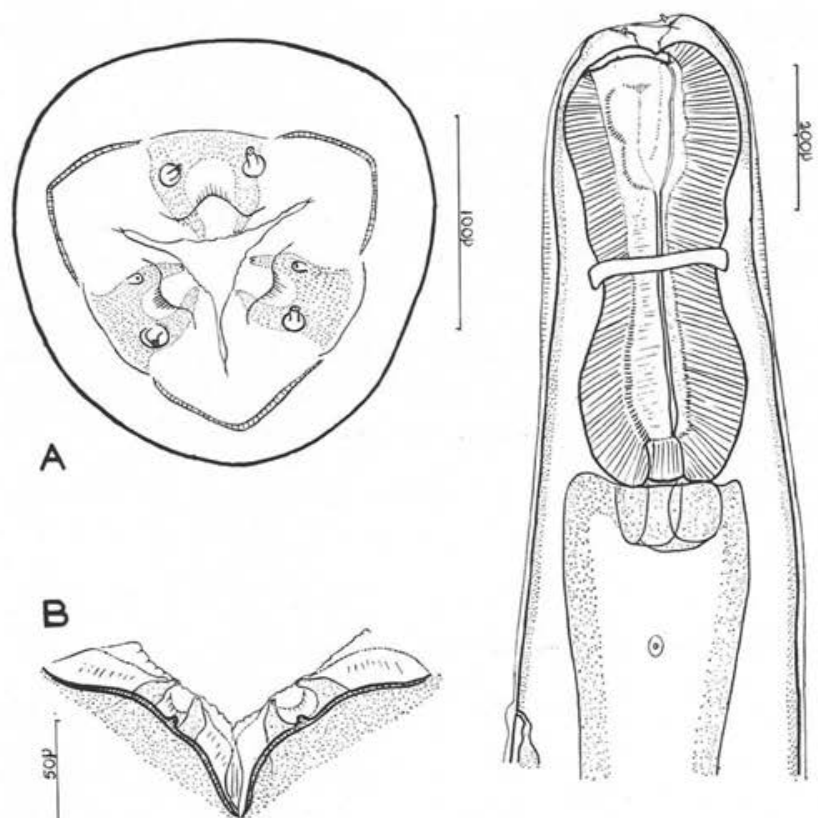

$E$
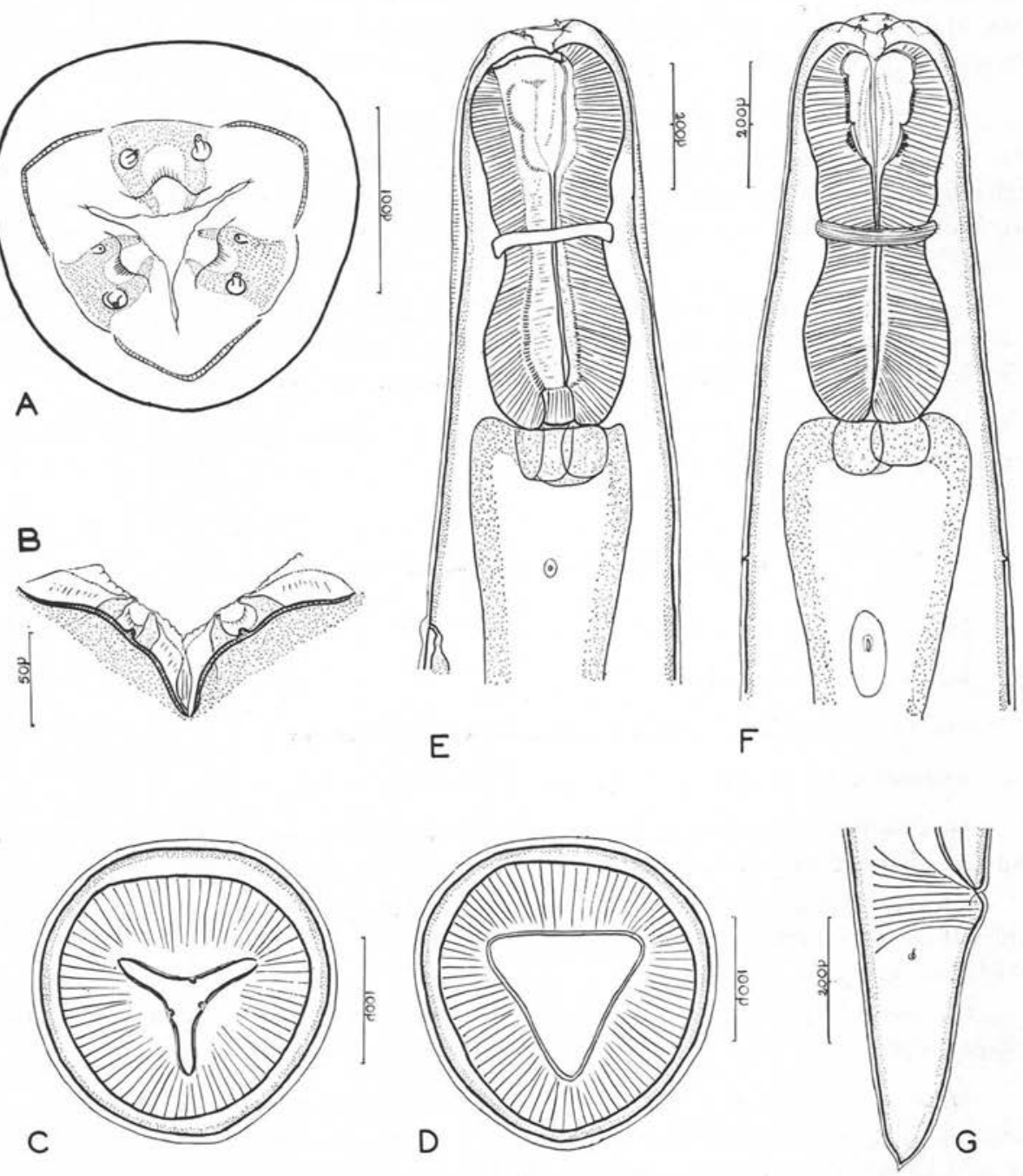

FIg. 3. - Chitwoodia chitwoodae n. g. n. sp. Femelle de 12,6 mm: A. Extrémité céphalique, vue apicale; B. Lèvres subventrales, face interne; C. Extrémité céphalique, coupe optique au niveau de la capsule buccale; D. Extrémité céphalique, coupe optique au niveau du début de l'œesophage; E. Extrémité antérieure, vue latérale; F. Extrémité antérieure, vue ventrale; G. Queue, vue latérale 
Femelle : Corps long de 12,6 mm et large de 0,5 mm. Esophage long de 0,62 $\mathrm{mm}$ et large de $0,25 \mathrm{~mm}$ au renflement antérieur, $0,16 \mathrm{~mm}$ au niveau de l'anneau nerveux et $0,23 \mathrm{~mm}$ au renflement postérieur. Anneau nerveux, diérides, pore excréteur respectivement à $0,34 \mathrm{~mm}$, à $0,88 \mathrm{~mm}$ et à $1,02 \mathrm{~mm}$ de l'apex.
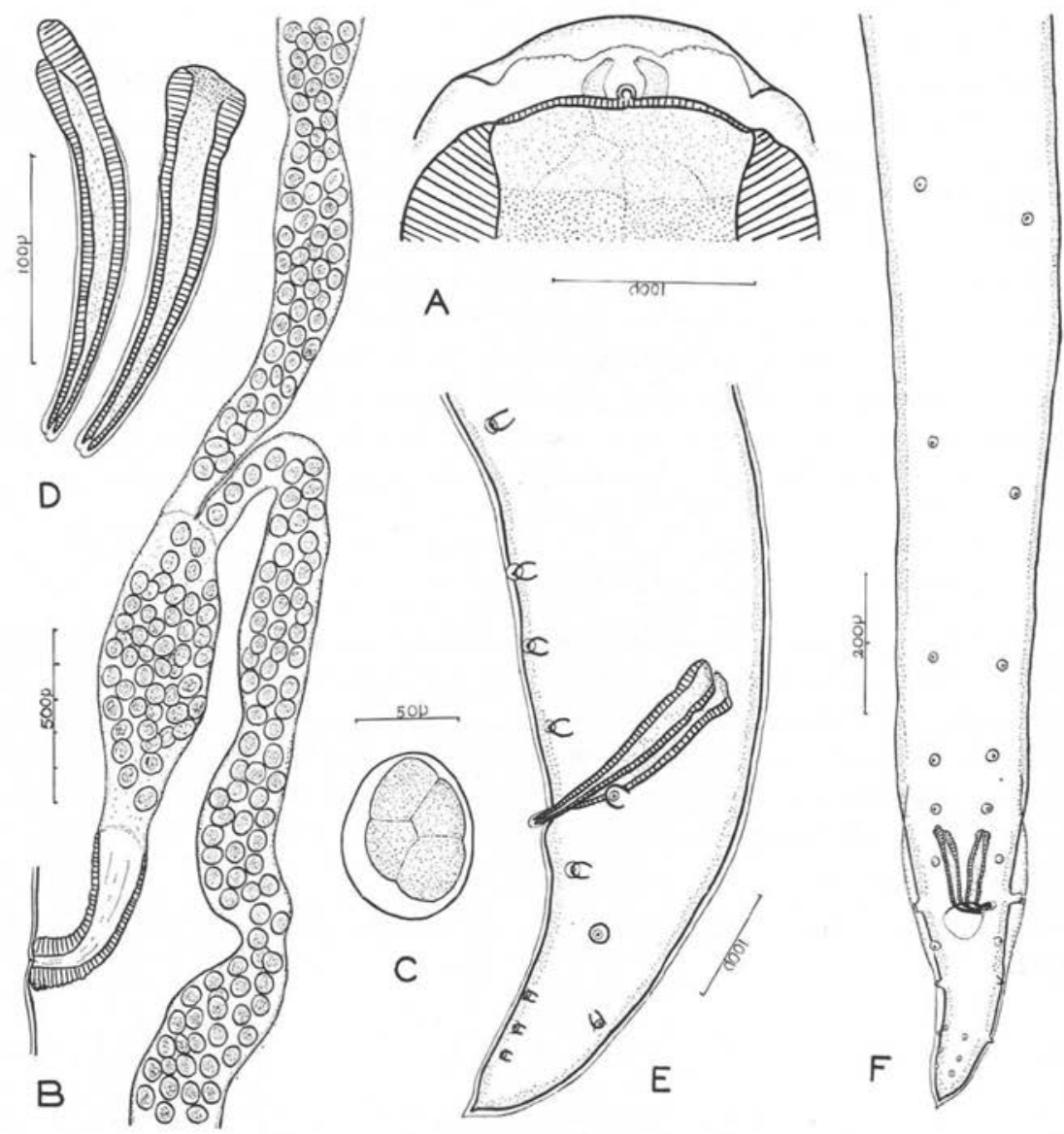

FIG. 4. - Chitwoodia chitwoodae n. g. n. sp. Femelle de 12,6 mm: A. Lèvre dorsale, face interne ; B. Appareil génital; C. Cuf. Mâle de 9,75 mm: D. Spicules; E. Queue, vue latérale; F. Extrémité postérieure, vue ventrale

Vulve située à $4,95 \mathrm{~mm}$ de l'extrémité postérieure. Vagin musculeux, dirigé en avant, long de $0,53 \mathrm{~mm}$, large de $0,15 \mathrm{~mm}$. Appareil génital de type amphidelphe (fig. 4, B). CEufs non embryonnés, à coque mince, mesurant 0,073-0,08 mm sur 0,055$0,062 \mathrm{~mm}$ (fig. 4, C). Queue longue de $0,47 \mathrm{~mm}$. Phasmides symétriques, situés à $0,35 \mathrm{~mm}$ de l'extrémité postérieure (fig. 3, G). 
Discussion : Les points les plus marquants du parasite résident dans :

- le cycle papillaire externe formé de 4 papilles simples et pédonculées ;

- la bouche formée de trois lèvres réduites, soudées à leurs bords latéraux et munies de pulpes labiales;

- l'œsophage simple et claviforme ;

- l'œuf non embryonné, à coque mince.

Nous sommes en présence d'un nématode très synthétique. La présence des puipes labiales évoque celles des Ascarides, mais si l'on admet l'importance du cycle papillaire céphalique et surtout de l'œsophage simple claviforme, il serait plus logique de le ranger dans les Seuratoidea, Chabaud, Campana-Rouget et Brygoo, 1959, à côté de Campanarougetia, Lê-van-Hoa et Pham-ngoc-Khuê, 1967.

Il se distingue des Seuratidae, Hall, 1916, Schneidernematidae, Freitas, 1956, Quimperidae, Gendre, 1926, Cucullanidae, Mueller, 1777 et Campanarougetidae (=Campanarougetinae, Lê-van-Hoa et Pham-ngoc-Khuê, 1967), Skrjabin et Ivashkin, 1968, par la présence des pulpes labiales. Nous proposons de créer pour lui une famille nouvelle pour laquelle nous proposons le nom Chitwoodidae n.f. en l'honneur du Professeur M. B. Chitwood. Les définitions de la famille et du genre sont confondues :

\section{Chitwoodidae n. f.}

Seuratoidea : Bouche entourée de 3 lèvres réduites et soudées à leurs bords latéraux. Pulpes labiales vestigiales. Cycle papillaire externe ayant 4 papilles simples et pédonculées. Capsule buccale faible mais chitinoide, munie de 3 petites dents. Esophage simple, court et trapu, dilaté antérieurement. Mâle: spicules courts et subégaux. Gubernaculum et ventouse précloacale absents. Ailes caudales étroites. Femelle : vulve postéquatoriale. Eufs non embryonnés et à coque mince. Parasites des poissons d'eau douce.

Genre unique: Chitwoodia n. g.

Espèce type: Chitwoodia chitwoodae n. sp. parasite de Puntius bramoides au Sud Viêt-nam.

\section{Remarques.}

1) En se basant d'une part sur la morphologie de Chitwoodia chitwoodae n. sp., Campanarougetia campanarougetae, Lê-van-Hoa et coll., 1967, et d'autre part sur celle des larves de Cucullanus chabaudi, Lê-van-Hoa et coll., 1967 (publication ultérieure), nous essayons d'établir une lignée Gendria-Chitwoodia-Campanarougetia-Cucullanus.

- Gendria, Baylis 1930 : Bouche circulaire ou hexagonale sans lèvres. Capsule buccale triangulaire munie de trois grosses dents. Esophage claviforme.

- Chitwoodia n. g. : Bouche formée de trois lèvres distinctes, mais réduites. Capsule buccale triangulaire munie de trois petites dents. Esophage claviforme et dilaté antérieurement. 
- Campanarougetia, Lê-vän-Hoa et Pham-ngoc-Khuê 1967: Bouche triangulaire sans lèvres. Capsule buccale triangulaire, sans dents. Esophage claviforme, très dilaté antérieurement.

- Cucullanus, Mueller 1777: Bouche et capsule buccale allongées dorso-ventralement. Esophage claviforme très dilaté antérieurement et muni de baguettes chitinoïdes.

2) Cette étude comparative permet de préciser la place des Cucullanus parmi les Seuratoidea, Chabaud, Campana et Brygoo, 1959. Inglis a raison d'écrire * The Cucullanidae is referred to the superfamily Seuratoidea, Ascaridida ».

Cependant, Skrjabin et Ivashkin (1968), utilisant en réalité les divisions d'Inglis, suppriment la superfamille et répartissent les espèces en Ascaridata, Spirurata, Cucullanata et Oxyurata, avec les Cucullanata (hyperspécialisés!) comme formes de passage entre les Ascarides et les Spirurides.

Cela revient à escamoter le problème posé par tout ce groupe qui peut précisément faire comprendre que!s sont les rapports entre les Ascarides et les Spirurides. Sans doute, faudrait-il attendre de nouvelles observations pour justifier les affinités des Seuratoidea, parasites synthétiques et si souvent difficiles à interpréter. Ainsi, Quentin conserve actuellement la classification d'Inglis mais replace les Echinonema Linstow, 1898, près des Seuratidae en démontrant les affinités avec les Rictulariidae (communication personnelle).

3) En ce qui concerne la désignation de cette superfamille, nous avons proposé en 1967, la dénomination Cucullanoidea (= Seuratoidea, Chabaud, Campana et Brygoo, 1959) puisque le taxon supragénérique le plus ancien est celui des Cucullanidae, Cobbold, 1864. Mais cela devient inextricable car Skrjabin et Ivashkin utilisent Cucullanoidea pour regrouper seulement les Cucullanidae, Quimperidae et Campanarougetidae. Il y aurait donc avantage, contrairement aux règles de la nomenclature, à reprendre la désignation Seuratoidea. Chabaud, Campana-Rouget et Brygoo, 1959.

\section{Bibliographie}

Bain (O.) et Philippon (B.), 1969. - Recherche sur des larves de Nématodes Ascaridida trouvées chez Simulium damnosum. Ann. Par., 44 (2), pp. 147-156.

Chabaud (A.-G.), in Grassé (P.-P.), 1965. - Traité de Zoologie. Tome IV, fasc. III, pp. 732 1497. Masson et $C^{\text {le }}$, Editeurs, Paris.

Chabaud (A.-G.), Campanarouget (Y.) et Brygoo (E.-R.), 1959. - Les Nématodes Seuratoidea, nov. sup. fam. et l'origine des Spirurida. C.R. Acad. Sc., CCXLVIII, pp. 14491451.

-, Campana Rouget (Y.) et Brygoo (E.-R.), 1960. - Les Nématodes Seuratoidea. Ann. Par., 35 (3), pp. 316-346.

INGLIS (W. G.), 1965. - Patterns of evolution in parasitic Nematode. Evolution of parasites. Third symposium of the British Society for Parasitology. Blackwell Scientific Publications, Oxford. 
—, 1967. - The relationship of the Nematodes Superfamilly Seuratoidea. J. Helm., XLI (2/3), pp. 115-136.

KARVE (J. N.), 1941. - Some nematodes parasites of fishes, I. J. Uni. Bombay, N.S., 10, pp. $9-42$.

—, 1944. - On a small collection of parasitic nematodes from Anura. Proc. Ind. Acad. Sc., 19 (3), pp. 71-77.

Le-Van-HoA et Pham-Ngoc-Khuê, $1967 a$. - Morphologie et Cycle évolutif de Cucullanus chabaudi n. sp., parasites de poissons Pangasius pangasius H.B. (P. Bucchani) du Sud Viêt-nam. Bull. Soc. Path. Exo., 60 (3), pp. 315-318.

—, 1967 b. - Relation entre les Seuratoidea et les Cucullans par l'intermédiaire d'un nouveau nématode Campanarougetia campanarougetae n. g. n. sp., parasite des Anguilles du Sud Viêt-nam. Ibid., 60 (4), pp. 393-398.

SKRJABIN (K. I.) et IvashKin (V. M.), 1968. - « On the elimination of the superfamily Seuratoidea Chabaud, Campana Rouget \& Brygoo, 1959, from the nematode classification ». Izv. Akad. Nauk. SSSR Ser. bio., n ${ }^{\circ}$ 6, pp. 789-792 (en russe).

YORKE (W.) et MAPLESTONE (P. A.), 1926. - The nematode parasites of vertebrates, $536 \mathrm{pp}$. J. et A. Churchill. London. 